International Journal of Difference Equations

ISSN 0973-6069, Volume 14, Number 2, pp. 127-148 (2019)

https://dx.doi.org/10.37622/IJDE/14.2.2019.127-148

\title{
Characterization of Higher-Order Neutral Difference Equations with Several Delays
}

\author{
N. Parhi \\ National Institute of Science Education and Research \\ Department of Mathematics \\ Bhubaneswar-751005, India
}

\author{
A. K. Tripathy \\ Sambalpur University \\ Department of Mathematics \\ Sambalpur-768019, India
}

\begin{abstract}
This paper is concerned with the characterization of the linear higher-order neutral difference equations of the form

$\left(E_{r}\right) \quad \Delta^{k}(y(n)-p y(n-s))+(-1)^{r} \sum_{i=1}^{m} q_{i} y\left(n-\sigma_{i}\right)=0, \quad r=1$ or 2

for $|p|<\infty$. Using the linearized oscillation method we have studied the oscillation of a class of nonlinear neutral difference equations of the form

$$
\Delta^{k}(y(n)-p(n) g(y(n-s)))+\sum_{i=1}^{m} q_{i}(n) h_{i}\left(y\left(n-\sigma_{i}\right)\right)=0
$$

with respect to its associated linear difference equations $\left(E_{r}\right)$.
\end{abstract}

AMS Subject Classifications: 39A10, 39A12.

Keywords: Oscillation, non-oscillation, neutral difference equation, linearized oscillation.

Received May 29, 2019; Accepted August 13, 2019

Communicated by Mehmet Ünal 


\section{Introduction}

Consider the higher-order neutral difference with several delays of the form

$\left(E_{r}\right) \quad \triangle^{k}(y(n)-p y(n-s))+(-1)^{r} \sum_{i=1}^{m} q_{i} y\left(n-\sigma_{i}\right)=0$,

where $\triangle$ is the forward difference operator defined by $\triangle y(n)=y(n+1)-y(n), q_{i} \geq 0$ such that $\sum_{i=1}^{m} q_{i}>0, \sigma_{i}>0, s>0$ are integers, $k \geq 1, r=1$ or 2 and $p \in \mathbb{R}$. Its characteristic equation is given by

$$
\left(C_{r}\right) \quad\left(1-p \lambda^{-s}\right)(\lambda-1)^{k}+(-1)^{r} \sum_{i=1}^{m} q_{i} \lambda^{-\sigma_{i}}=0 .
$$

$\left(E_{r}\right)$ may be treated as the discrete analogue of

$$
(y(t)-p y(t-\tau))^{(k)}+(-1)^{r} \sum_{i=1}^{m} q_{i} y\left(t-\sigma_{i}\right)=0
$$

whose characteristic equation is given by

$$
\lambda^{k}\left(1-p e^{-\lambda r}\right)+(-1)^{r} \sum_{i=1}^{m} q_{i} e^{-\lambda \sigma_{i}}=0 .
$$

Very often the study of difference equations can similarly be dealt with the differential equations. However, the study of characteristic equations of type $\left(C_{r}\right)$ and (1.1) are altogether a different approach. Because $\left(C_{r}\right)$ is an algebraic equation of degree $(k+s+\sigma)$, where $\sigma=\max \left\{\sigma_{i}: 1 \leq i \leq m\right\}$, given an equation which depends on several parameters, it is desirable to find the exact dependence of the qualitative behaviour of the set of solutions of this equation on these parameters (see, e.g., [3,9]). In this paper, we will be concerned with $\left(E_{r}\right)$ which depends on $(2 m+3)$ parameters.

The primary objective of this work is to study $\left(C_{r}\right)$ and investigate the oscillatory behaviour of solutions of $\left(E_{r}\right)$ which is set out with $(2 m+3)$ parameters. We keep our base on the underlined proposition whose proof is very much similar to the proof of [4, Theorem 7.1.1].

Proposition A. Every solution of $\left(E_{r}\right)$ is oscillatory if and only if $\left(C_{r}\right)$ has no positive real roots.

In order to meet our secondary goal, an attempt is made here to study the nonlinear difference equations of the form

$$
\triangle^{k}(y(n)-p(n) g(y(n-s)))+\sum_{i=1}^{m} q_{i}(n) h_{i}\left(y\left(n-\sigma_{i}\right)\right)=0,
$$

where $g, h_{i} \in C(\mathbb{R}, \mathbb{R}), 1 \leq i \leq m$ such that

$$
\limsup _{n \rightarrow \infty} p(n)=p_{0} \in[1, \infty)
$$




$$
\begin{aligned}
& \lim _{n \rightarrow \infty} q_{i}(n)=q_{i}^{0} \in(0, \infty), \\
& \frac{q(u)}{u} \geq 1, \quad u \neq 0 \quad \text { and } \quad \lim _{|u| \rightarrow \infty} \frac{q(u)}{u}=1, \\
& u h_{i}(u)>0, u \neq 0 ; \quad\left|h_{i}(u)\right| \geq h_{i}^{0}, \text { for }|u| \geq \lambda_{0}>0
\end{aligned}
$$

and

$$
\lim _{|u| \rightarrow \infty} \frac{h_{i}(u)}{u}=1, \quad 1 \leq i \leq m
$$

We may note that (1.2) is highly nonlinear and hence it is very difficult to predict the behaviour of its solutions. In this work, we study the oscillatory behaviour of solutions of (1.2) with the help of the oscillatory behaviour of solutions of its limiting equation

$$
\triangle^{k}\left(x(n)-p_{0} x(n-s)\right)+\sum_{i=1}^{m} q_{i}^{0} x\left(n-\sigma_{i}\right)=0
$$

by using the linearization technique. In this direction, we refer the reader to some of the works $[4,7,8,10]$.

There are several results (see, e.g., $[1,2,11]$ ) concerning the sufficient conditions for oscillation of all solutions of the higher-order nonlinear neutral delay differential/difference equations. However, the study of such equations via its limiting equation has received less attention. There exist some examples (see, e.g., [6]), where the study of appropriate linear equations predicts the dynamics of nonlinear ones. In stability theory of differential and difference equations, the linearization technique plays an important role. By linearized oscillation we mean that the certain nonlinear differential/difference equations under appropriate hypotheses have the same oscillatory behaviour as associated linear equations $[7,8,10]$. By a solution of $\left(E_{r}\right) /(1.2)$ we understand a real valued function $y(n)$ defined on $N(-\rho)=\{-\rho,-\rho+1, \ldots\}$ which satisfies $\left(E_{r}\right) /(1.2)$, where $\rho=\max \left\{s, \sigma_{i}: 1 \leq i \leq m\right\}$. If

$$
y(n)=\phi_{n}, \quad n=-\rho,-\rho+1, \ldots, 0,1,2, \ldots
$$

are given, then $\left(E_{r}\right) /(1.2)$ admits a unique solution satisfying the initial conditions (1.8). As is customary, a solution $y(n)$ of $\left(E_{r}\right) /(1.1)$ is said to be oscillatory, if it is neither eventually positive nor eventually negative. Otherwise, it is called nonoscillatory.

\section{Oscillation Results for $\left(E_{2}\right)$}

To facilitate the discussion, we shall begin with Proposition A. We may note that

$$
\triangle^{k}(y(n)-p y(n-s))=\sum_{j=0}^{k}(-1)^{j}[y(n+k-j)-p y(n+k-j-s)]\left(\begin{array}{c}
k \\
j
\end{array}\right)
$$


(see, e.g., [5, p.16]). Hence, $\left(E_{r}\right)$ can be put in the form [4, (7.1.1)] with matrices as the real numbers. Since we are concerned with positive roots of $\left(C_{r}\right)$, our attention is restricted to the case when $\lambda>0$.

Theorem 2.1. Assume that $0<s \leq \sigma_{1} \leq \sigma_{2} \leq \ldots \leq \sigma_{m}$ and $0 \leq p \leq 1$. If

i) $\quad \sum_{i=1}^{m} q_{i} \geq(1-p)\left(1-p^{1 / s}\right)^{k}$, when $k$ is odd

ii) $\quad \sum_{i=1}^{m} q_{i} \geq p$, when $k$ is even

hold, then $\left(E_{2}\right)$ is oscillatory.

Proof. It is enough to show that $\left(C_{2}\right)$ has no positive real roots. If possible, let $\lambda_{0}>0$ be a root of $\left(C_{2}\right)$. The contradiction is obvious if $\lambda_{0}=1$. Hence, $\lambda_{0}>1$ or $<1$. Since we can write $\left(C_{2}\right)$ as

$$
\lambda_{0}^{\sigma_{1}}\left(\lambda_{0}^{s}-p\right)\left(\lambda_{0}-1\right)^{k}+\lambda_{0}^{s} \sum_{i=1}^{m} q_{i} \lambda_{0}^{\sigma_{1}-\sigma_{i}}=0,
$$

then $\lambda_{0}>1$ implies that

$$
0<\lambda_{0}^{s} \sum_{i=1}^{m} q_{i} \lambda_{0}^{-\sigma_{i}}=-\left(\lambda_{0}^{s}-p\right)\left(\lambda_{0}-1\right)^{k}<0,
$$

a contradiction. Thus $0<\lambda_{0}<1$. If $\lambda_{0}=p^{1 / s}$, then (2.1) leads to a contradiction. Consequently, it follows that $\lambda_{0}>p^{1 / s}$ or $0<\lambda_{0}<p^{1 / s}$. Let $k$ be odd. If $\lambda_{0}<p^{1 / s}$, then there is a contradiction with (2.2). Thus $1>\lambda_{0}>p^{1 / s}$. From (2.1), it follows that

$$
\sum_{i=1}^{m} q_{i} \lambda_{0}^{\sigma_{1}-\sigma_{i}}=\lambda_{0}^{\sigma_{1}-s}\left(\lambda_{0}^{s}-p\right)\left(1-\lambda_{0}\right)^{k}
$$

due to odd $k$. Using the fact that $\left(1-\lambda_{0}\right)^{k}<\left(1-p^{1 / s}\right)^{k}$, we obtain from (2.3) that

$$
\begin{aligned}
\sum_{i=1}^{m} q_{i} \leq \sum_{i=1}^{m} q_{i} \lambda_{0}^{\sigma_{1}-\sigma_{i}} & =\lambda_{0}^{\sigma_{1}-s}\left(\lambda_{0}^{s}-p\right)\left(1-\lambda_{0}\right)^{k} \\
& <\left(\lambda_{0}^{s}-p\right)\left(1-\lambda_{0}\right)^{k}<(1-p)\left(1-p^{1 / s}\right)^{k},
\end{aligned}
$$

a contradiction.

Next, we suppose that $k$ is even. Since (2.1) can be written as

$$
\sum_{i=1}^{m} q_{i} \lambda_{0}^{\sigma_{1}-\sigma_{i}}=\lambda_{0}^{\sigma_{1}-s}\left(p-\lambda_{0}^{s}\right)\left(\lambda_{0}-1\right)^{k}
$$


then $p-\lambda_{0}^{s}<0$ gives a contradiction to (2.4). Hence, $0<\lambda_{0}<p^{1 / s}<1$ and (2.4) yields

$$
\begin{aligned}
\sum_{i=1}^{m} q_{i} \leq \sum_{i=1}^{m} q_{i} \lambda_{0}^{\sigma_{1}-\sigma_{i}} & =\lambda_{0}^{\sigma_{1}-s}\left(p-\lambda_{0}^{s}\right)\left(\lambda_{0}-1\right)^{k} \\
& <\left(p-\lambda_{0}\right)\left(\lambda_{0}-1\right)^{k}<p\left(\lambda_{0}-1\right)^{k}<p,
\end{aligned}
$$

a contradiction. Consequently, $\left(E_{2}\right)$ is oscillatory by Proposition A.

Remark 2.2. It seems that we can drop the condition $\sigma_{1}<\sigma_{2}<\ldots<\sigma_{m}$. If not, let $\sigma_{j}=\max \left\{\sigma_{i}: 1 \leq i \leq m\right\}$. Then $\left(E_{2}\right)$ is of degree $\sigma_{j}+k-s$. If $\sigma_{l}=\min \left\{\sigma_{i}: 1 \leq\right.$ $i \leq m\}$, then we may use $\sigma_{l}$ in the place of $\sigma_{1}$. In fact, since $\sigma_{1}, \sigma_{2}, \ldots, \sigma_{m}$ are finite in numbers, we can always put these in increasing order. Hence without loss of generality, we can take $\sigma_{1} \leq \sigma_{2} \leq \ldots \leq \sigma_{m}$.

Theorem 2.3. Let $p=1$. Then $\left(E_{2}\right)$ is oscillatory, if $k$ is odd and $k$ is even when $0<s \leq \sigma_{1}$ and $\sum_{i=1}^{m} q_{i} \geq 1$.

Proof. For $p=1$, Eq. $\left(C_{2}\right)$ takes the form

$$
\left(1-\lambda_{0}^{-s}\right)\left(\lambda_{0}-1\right)^{k}+\sum_{i=1}^{m} q_{i} \lambda_{0}^{-\sigma_{i}}=0
$$

when we assume that $\lambda_{0}$ is a root of $\left(C_{2}\right)$. We need to show that $\left(C_{2}\right)$ doesn't admit a positive root. Assume that $\lambda_{0}>0$ is a root of $\left(C_{2}\right)$ such that $\lambda_{0} \neq 1$. Then $0<\lambda_{0}<1$ and hence (2.5) becomes

$$
\begin{aligned}
0<\sum_{i=1}^{m} q_{i} \lambda_{0}^{-\sigma_{i}} & =-\left(1-\lambda_{0}^{-s}\right)\left(\lambda_{0}-1\right)^{k} \\
& =-\left(\lambda_{0}^{-s}-1\right)\left(1-\lambda_{0}\right)^{k}<0,
\end{aligned}
$$

when $k$ is odd, a contradiction. Let $k$ be even. For $\lambda_{0}<1$, we obtain from (2.5) that

$$
\begin{aligned}
\sum_{i=1}^{m} q_{i}<\sum_{i=1}^{m} q_{i} \lambda_{0}^{\sigma_{1}-\sigma_{i}} & =\lambda_{0}^{\sigma_{1}-s}\left(1-\lambda_{0}^{s}\right)\left(1-\lambda_{0}\right)^{k} \\
& \leq\left(1-\lambda_{0}^{s}\right)\left(1-\lambda_{0}\right)^{k}<1
\end{aligned}
$$

a contradiction. Hence $\lambda_{0}$ is not a positive root of $\left(C_{2}\right)$. This completes the proof.

Theorem 2.4. Let $p>1$. If

(i) $\sum_{i=1}^{m} q_{i} \geq p(p-1)^{k}$ and $\sigma_{1} \leq \sigma_{2} \leq \ldots \leq \sigma_{m} \leq s$ when $k$ is odd, 
(ii) $\sum_{i=1}^{m} q_{i} \geq \max \left\{p(p-1)^{k}, p\right\}$ and $\sigma_{1} \leq \sigma_{2} \leq \ldots \leq \sigma_{m} \leq s \leq k$ when $k$ is even, then $\left(E_{2}\right)$ is oscillatory.

Proof. It is sufficient to show that $\left(C_{2}\right)$ has no positive roots. If $\lambda_{0}$ is a positive root of $\left(C_{2}\right)$, then either $\lambda_{0}>1$ or $\lambda_{0}<1$. For $\lambda_{0}>1$, we raise two cases viz., $(i) 1<\lambda_{0}<p$ and $(i i) 1<p \leq \lambda_{0}$. Let the latter case hold. Then $\lambda_{0}^{s}>p$ implies that (2.2) yield a contradiction. Also, we have same contradiction when $\lambda_{0}^{s} \geq p$. Consequently, $\lambda_{0}^{s}<p$. Since

$$
\lambda_{0}^{\sigma_{m}}\left(\lambda_{0}^{s}-p\right)\left(\lambda_{0}-1\right)^{k}+\lambda_{0}^{s} \sum_{i=1}^{m} q_{i} \lambda_{0}^{\sigma_{m}-\sigma_{i}}=0
$$

then

$$
\begin{aligned}
\sum_{i=1}^{m} q_{i} \leq \sum_{i=1}^{m} q_{i} \lambda_{0}^{\sigma_{m}-\sigma_{i}} & =-\lambda_{0}^{\sigma_{m}-s}\left(\lambda_{0}^{s}-p\right)\left(\lambda_{0}-1\right)^{k} \\
& \leq\left(p-\lambda_{0}^{s}\right)\left(\lambda_{0}-1\right)^{k}<p(p-1)^{k}
\end{aligned}
$$

a contradiction due to odd $k$. Let $\lambda_{0}<1$. Then $0<\lambda_{0}<1<p$ implies that $\lambda_{0}^{s}<1<p$ and hence

$$
0<\lambda^{s} \sum_{i=1}^{m} q_{i} \lambda_{0}^{-\sigma_{i}}=-\left(\lambda_{0}^{s}-p\right)\left(\lambda_{0}-1\right)^{k}<0
$$

due to odd $k$, a contradiction. Assume that $k$ is even. Using the fact that $\lambda_{0}^{\sigma_{i}-\sigma_{m}}<1$, we obtain

$$
\begin{aligned}
\sum_{i=1}^{m} q_{i}<\sum_{i=1}^{m} q_{i} \lambda_{0}^{-\sigma_{i}} & =\lambda_{0}^{-s}\left(p-\lambda_{0}^{s}\right)\left(\lambda_{0}-1\right)^{k} \\
& =\lambda_{0}^{k-s}\left(p-\lambda_{0}^{s}\right)\left(1-\frac{1}{\lambda_{0}}\right)^{k}<p, \text { if } k>s
\end{aligned}
$$

a contradiction. Hence, $\left(C_{2}\right)$ has no positive roots. The proof of the theorem is complete.

Theorem 2.5. Let $p \leq 0$. Assume that

$$
\sum_{i=1}^{m} q_{i} \geq 1-p>-p, \quad s \leq \sigma_{1} \leq \sigma_{2} \leq \ldots \leq \sigma_{m}
$$

holds, when $k$ is odd. Then $\left(E_{2}\right)$ is oscillatory. 
Proof. If possible, let $\lambda_{0}>0$ be a root of $\left(C_{2}\right)$. From (2.1), it follows that

$$
\sum_{i=1}^{m} q_{i} \lambda_{0}^{s-\sigma_{i}}=\left(\lambda_{0}^{s}-p\right)\left(1-\lambda_{0}\right)^{k} .
$$

Then for odd $k$ and $0<\lambda_{0}<1$, we obtain that

$$
\sum_{i=1}^{m} q_{i} \leq \sum_{i=1}^{m} q_{i} \lambda_{0}^{s-\sigma_{i}}=\left(\lambda_{0}^{s}-p\right)\left(1-\lambda_{0}\right)^{k}<1-p,
$$

a contradiction. Suppose that $\lambda_{0}>1$. Then

$$
0<\sum_{i=1}^{m} q_{i} \lambda_{0}^{s-\sigma_{i}}=\left(\lambda_{0}^{s}-p\right)\left(1-\lambda_{0}\right)^{k}<0,
$$

which is absurd. Let $k$ be even. Therefore, $\lambda_{0}>0$ implies that

$$
0<\sum_{i=1}^{m} q_{i} \lambda_{0}^{s-\sigma_{i}}=-\left(\lambda_{0}^{s}-p\right)\left(\lambda_{0}-1\right)^{k}<0,
$$

a contradiction. Thus by Proposition A, $\left(E_{2}\right)$ is oscillatory. This completes the proof.

Theorem 2.6. If $k$ is even, $p>0$ and $\sigma_{1}<\sigma_{2}<\ldots<\sigma_{m}<s$, then $\left(E_{2}\right)$ admits $a$ positive solution which tends to zero as $n \rightarrow \infty$.

Proof. We consider

$$
F(\lambda)=\left(1-p \lambda^{-s}\right)(\lambda-1)^{k}+\sum_{i=1}^{m} q_{i} \lambda^{-\sigma_{i}}=0 .
$$

Clearly, $F(1)>0$. Further,

$$
\begin{aligned}
F(\lambda) & =\frac{1}{\lambda^{\sigma_{m}}}\left[\lambda^{\sigma_{m}}\left(1-p \lambda^{-s}\right)(\lambda-1)^{k}+\sum_{i=1}^{m} q_{i} \lambda^{\sigma_{m}-\sigma_{i}}\right] \\
& =\lambda^{-\sigma_{m}}\left[-\left(p \lambda^{-\left(s-\sigma_{m}\right)}-\lambda^{\sigma_{m}}\right)(\lambda-1)^{k}+\sum_{i=1}^{m} q_{i} \lambda^{\sigma_{m}-\sigma_{i}}\right] \\
& \rightarrow-\infty \text { as } \lambda \rightarrow 0+
\end{aligned}
$$

implies that there exists $1>\lambda_{0}>0$ such that $F\left(\lambda_{0}\right)=0$. Consequently, $\left\{\lambda_{0}^{n}\right\}$ is a positive solution of $\left(E_{2}\right)$ which tends to zero as $n \rightarrow \infty$.

Theorem 2.7. If $k$ is odd, $p<0$ and $\sigma_{1}<\sigma_{2}<\ldots<\sigma_{m}<s$, then ( $\left.E_{2}\right)$ admits a positive solution which tends to zero as $n \rightarrow \infty$. 
Proof. The proof of the theorem follows from the proof of Theorem 2.6. Hence the details are omitted.

Theorem 2.8. Let $0 \leq p<1$ and $k$ be odd. If

$$
\sum_{i=1}^{m}\left(\sigma_{i}\right)^{-\sigma_{i}}\left(k+\sigma_{i}\right)^{\left(k+\sigma_{i}\right)} q_{i}>(1-p) k^{k},
$$

then $\left(E_{2}\right)$ is oscillatory.

Proof. Suppose on the contrary that $\lambda>0$ is a root of $\left(C_{2}\right)$. With $F(\lambda)$ as in (2.6), we have $F(1)>0$ and $F(\infty)=\infty$. In what follows, $F(\lambda)$ has positive roots in $(0,1) \cup(1, \infty)$. Assume that $0<\lambda<1$. Then $F(\lambda)$ becomes

$$
F(\lambda)>(1-\lambda)^{k}\left[-(1-p)+\sum_{i=1}^{m} q_{i} \frac{\lambda^{-\sigma_{i}}}{(1-\lambda)^{k}}\right],
$$

where $\lambda<1$ implies $\left(1-p \lambda^{-s}\right)<1-p$. Set $f(\lambda)=\frac{\lambda^{-\sigma_{i}}}{(1-\lambda)^{k}}$. Then $f^{\prime}(\lambda)=0$ implies that $\lambda=\frac{\sigma_{i}}{k+\sigma_{i}}$. It follows from

$$
f^{\prime \prime}(\lambda)=\frac{\sigma_{i} \lambda^{-\left(2+\sigma_{i}\right)}}{(1-\lambda)^{k+1}}\left[(1-\lambda)\left(1+\sigma_{i}\right)-k \lambda\right]+\frac{k \lambda^{-\left(1+\sigma_{i}\right)}}{(1-\lambda)^{k+2}}\left[\lambda(k+1)-\sigma_{i}(1-\lambda)\right]
$$

that $f^{\prime \prime}(\lambda)>0$, for $\lambda=\frac{\sigma_{i}}{\left(k+\sigma_{i}\right)}$. Hence, $\min f(\lambda)$ exists at $\lambda=\frac{\sigma_{i}}{\left(k+\sigma_{i}\right)}$. Consequently,

$$
\min f(\lambda)=\frac{\left(k+\sigma_{i}\right)^{k+\sigma_{i}}}{\left(\sigma_{i}\right)^{\sigma_{i}} k^{k}}
$$

implies that

$$
F(\lambda)>(1-\lambda)^{k}\left[-(1-p)+\sum_{i=1}^{m} q_{i} \frac{\left(k+\sigma_{i}\right)^{k+\sigma_{i}}}{\left(\sigma_{i}\right)^{\sigma_{i}} k^{k}}\right]>0
$$

if and only if (2.7) holds.

Next, we suppose that $\lambda>1$. Therefore, $1-\lambda^{-s} p>1-p$ and

$$
F(\lambda)>(\lambda-1)^{k}\left[(1-p)+\sum_{i=1}^{m} q_{i} \frac{\lambda^{-\sigma_{i}}}{(\lambda-1)^{k}}\right] .
$$

If we set $f_{i}(\lambda)=\frac{\lambda^{-\sigma_{i}}}{(\lambda-1)^{k}}$, then it follows that $f_{i}^{\prime}(\lambda)<0$ and $f_{i}^{\prime \prime}(\lambda)>0$ for $\lambda>1$. Hence $\liminf _{\lambda \rightarrow \infty} f_{i}(\lambda)$ exists. Let $f_{i}^{0}=\liminf _{\lambda \rightarrow \infty} f_{i}(\lambda), f_{i}^{0} \in[0, \infty)$. Then (2.8) reduces to

$$
F(\lambda)>(\lambda-1)^{k}\left[(1-p)+\sum_{i=1}^{m} q_{i} f_{i}^{0}\right]>0 .
$$


In what follows, $f(\lambda)$ has no positive roots in $(0,1) \cup(1, \infty)$. By Proposition $\mathrm{A},\left(E_{2}\right)$ is oscillatory. Hence the theorem is proved.

\section{Oscillation Results for $\left(E_{1}\right)$}

In this section, the oscillatory behaviour of solutions of $\left(E_{1}\right)$ is studied with its characteristic equation $\left(C_{1}\right)$.

Theorem 3.1. Let $0 \leq p<1$ and $0<s<\sigma_{1}<\sigma_{2}<\ldots<\sigma_{m}$. Assume that any one of the following conditions

i) $\quad \sum_{i=1}^{m} q_{i} \geq p^{-1}(1-p)\left(1-p^{1 / s}\right)^{k}$,

ii) $\quad \sum_{i=1}^{m} q_{i} \geq p$

iii) $\quad \sum_{i=1}^{m} q_{i} \leq(1-p) p^{k}$

iv) $\quad \sum_{i=1}^{m} q_{i} \geq\left[(1+p)^{s}-p\right] p^{k}$

holds. Then $\left(E_{1}\right)$ is oscillatory.

Proof. It is sufficient to show that $\left(C_{1}\right)$ has no positive roots. If possible, let $\lambda_{0}>0$ be a root of $\left(C_{1}\right)$. We can write $\left(C_{1}\right)$ as follows.

$$
\sum_{i=1}^{m} q_{i} \lambda^{-\sigma_{i}}=\lambda^{-s}\left(\lambda^{s}-p\right)(\lambda-1)^{k}
$$

Indeed, $\lambda_{0}=1$ is not possible. Hence, either $0<\lambda_{0}<1$ or $\lambda_{0}>1$ holds. Suppose that $0<\lambda_{0}<1$ and $k$ is even. If $\lambda_{0}=p^{1 / s}$, then we have a contradiction from (3.1). Consequently, we raise two cases viz., $\lambda_{0}<p^{1 / s}$ and $\lambda_{0}>p^{1 / s}$. If the former holds, then it happens that

$$
0<\sum_{i=1}^{m} q_{i} \lambda_{0}^{-\sigma_{i}}=\lambda_{0}^{-s}\left(\lambda_{0}^{s}-p\right)\left(\lambda_{0}-1\right)^{k}<0
$$

which is absurd. With the latter case, it follows from (3.1) that

$$
\begin{aligned}
\sum_{i=1}^{m} q_{i}<\sum_{i=1}^{m} q_{i} \lambda_{0}^{-\sigma_{i}} & =\lambda_{0}^{-s}\left(\lambda_{0}^{s}-p\right)\left(\lambda_{0}-1\right)^{k} \\
& <p^{-1}(1-p)\left(1-p^{1 / s}\right)^{k}
\end{aligned}
$$

a contradiction. 
Let $k$ be odd. If $1>\lambda_{0}>p^{1 / s}$, then it is easy to obtain a contradiction from (3.1). Consider that $\lambda_{0}<p^{1 / s}$. Hence, $\sigma_{1}<\sigma_{2}<\ldots<\sigma_{m}$ implies that

$$
\sum_{i=1}^{m} q_{i}<\sum_{i=1}^{m} q_{i} \lambda_{0}^{\sigma_{1}-\sigma_{i}}=-\lambda_{0}^{\sigma_{1}-s}\left(p-\lambda_{0}^{s}\right)\left(\lambda_{0}-1\right)^{k}<p
$$

a contradiction.

Next, we assume that $\lambda_{0}>1$. We restrict our attention for the cases $\lambda_{0} \geq 1+p$ and $\lambda_{0}<1+p$. If $\lambda_{0} \geq 1+p$, then (3.1) yields

$$
\begin{aligned}
\sum_{i=1}^{m} q_{i}>\sum_{i=1}^{m} q_{i} \lambda_{0}^{-\sigma_{i}} & =\left(1-p \lambda_{0}^{-s}\right)\left(\lambda_{0}-1\right)^{k} \\
& >(1-p) p^{k},
\end{aligned}
$$

which is a contradiction. Thus, $\lambda_{0}<1+p$. Consequently, (3.1) becomes

$$
\begin{aligned}
\sum_{i=1}^{m} q_{i}(1+p)^{-\sigma_{i}}<\sum_{i=1}^{m} q_{i} \lambda_{0}^{-\sigma_{i}} & =\lambda_{0}^{-s}\left(\lambda_{0}^{s}-p\right)\left(\lambda_{0}-1\right)^{k} \\
& <\left[(1+p)^{s}-p\right] p^{k}
\end{aligned}
$$

which is a contradiction. From Proposition A, it concludes that $\left(E_{1}\right)$ is oscillatory. This completes the proof.

Theorem 3.2. Let $p=1$ and $s \leq \sigma_{1}<\sigma_{2}<\ldots<\sigma_{m}$. Assume that any one of the following conditions

i) $\quad \sum_{i=1}^{m} q_{i} \geq 1$

ii) $\quad \sum_{i=1}^{m} q_{i}<2^{s}-1$,

iii) $\quad \sum_{i=1}^{m} q_{i} \geq 2^{s}-1$

holds. Then every solution of $\left(E_{1}\right)$ oscillates.

Proof. Proceeding as in the proof of Theorem 3.1, we consider the cases of $\lambda_{0}<1$ and $\lambda_{0}>1$. Let $\lambda_{0}<1$ and the contradiction is obvious from (3.1) when $k$ is even. If $k$ is odd, then (3.1) yields

$$
\sum_{i=1}^{m} q_{i} \leq \sum_{i=1}^{m} q_{i} \lambda_{0}^{s-\sigma_{i}}=\left(1-\lambda_{0}^{-s}\right)\left(1-\lambda_{0}\right)^{k}<1
$$

which is a contradiction. Let $\lambda_{0}>1$. We restrict our attention for the cases $\lambda_{0} \geq 2$ and $1<\lambda_{0}<2$. If $\lambda_{0} \geq 2$, then $s \leq \sigma_{1}<\sigma_{2}<\ldots<\sigma_{m}$ implies that

$$
\sum_{i=1}^{m} q_{i} \geq \sum_{i=1}^{m} q_{i} \lambda_{0}^{s-\sigma_{i}} \geq 2^{s}-1
$$


a contradiction. Hence, $1<\lambda_{0}<2$. From (3.1), we obtain that

$$
\begin{aligned}
\sum_{i=1}^{m} q_{i} 2^{-\sigma_{i}}<\sum_{i=1}^{m} q_{i} \lambda_{0}^{-\sigma_{i}} & =\lambda_{0}^{-s}\left(\lambda_{0}^{s}-1\right)\left(\lambda_{0}-1\right)^{k} \\
& <2^{s}-1,
\end{aligned}
$$

a contradiction. The conclusion of the theorem follows from Proposition A. Hence, the theorem is proved.

Theorem 3.3. Let $p>1$ and $s \leq \sigma_{1}<\sigma_{2}<\ldots<\sigma_{m}$. Suppose that any one of the following conditions
i) $\quad \sum_{i=1}^{m} q_{i} \geq p$
ii) $\quad \sum_{i=1}^{m} q_{i}<\left(p^{s}-p\right)(p-1)^{k}$
iii) $\quad \sum_{i=1}^{m} q_{i} p^{-\sigma_{i}} \geq\left(p^{s}-p\right)(p-1)^{k}$

holds. Then $\left(E_{1}\right)$ is oscillatory.

Proof. Proceeding as in the proof of Theorem 3.2, we consider the case $\lambda_{0}<1$ when $k$ is odd. Therefore,

$$
\sum_{i=1}^{m} q_{i} \leq \sum_{i=1}^{m} q_{i} \lambda_{0}^{s-\sigma_{i}}=\left(p-\lambda_{0}^{-s}\right)\left(1-\lambda_{0}\right)^{k}<p
$$

gives a contradiction. Let $\lambda_{0}>1$. Then, we suppose two cases viz., $1<\lambda_{0}<p$ and $\lambda_{0} \geq p$. If the latter holds, then $s \leq \sigma_{1}<\sigma_{2}<\ldots<\sigma_{m}$ implies that

$$
\sum_{i=1}^{m} q_{i} \geq \sum_{i=1}^{m} q_{i} \lambda_{0}^{s-\sigma_{i}}=\left(p^{s}-p\right)(p-1)^{k}
$$

a contradiction to our assumption. Assume that the former holds. Then

$$
\begin{aligned}
\sum_{i=1}^{m} q_{i} p^{-\sigma_{i}}<\sum_{i=1}^{m} q_{i} \lambda_{0}^{-\sigma_{i}} & =\lambda_{0}^{-s}\left(\lambda_{0}^{s}-p\right)\left(\lambda_{0}-1\right)^{k} \\
& <\left(\lambda_{0}^{s}-p\right)\left(\lambda_{0}-1\right)^{k}<\left(p^{s}-p\right)(p-1)^{k}
\end{aligned}
$$

gives a contradiction to our assumption. This completes the proof of the theorem.

Theorem 3.4. Let $p<0$ and $s \leq \sigma_{1}<\sigma_{2}<\ldots<\sigma_{m}$. If any one of the following conditions

i) $\quad \sum_{i=1}^{m} q_{i} \geq 1-p$ 
ii) $\quad \sum_{i=1}^{m} q_{i}<(-p)^{k}(1-p)$,

iii) $\quad \sum_{i=1}^{m} q_{i}(1-p)^{s-\sigma_{i}} \geq(-p)^{k}\left[(1-p)^{s}-p\right]$

holds, then $\left(E_{1}\right)$ is oscillatory.

Proof. For our assertion, it is enough to show that there is no positive root of the type $\lambda_{0}<1$ or $\lambda_{0}>1$ for (3.1). The contradiction is obvious when $\lambda_{0}<1$ and $k$ is odd. Let $k$ be even. Using the fact that $s \leq \sigma_{1}<\sigma_{2}<\ldots<\sigma_{m}$, we obtain from (3.1) that

$$
\sum_{i=1}^{m} q_{i} \leq \sum_{i=1}^{m} q_{i} \lambda_{0}^{s-\sigma_{i}}=\left(\lambda_{0}^{-s}-p\right)\left(\lambda_{0}-1\right)^{k}<1-p
$$

which is a contradiction. Suppose that $\lambda_{0}>1$. Here, we predict two cases viz., $\lambda_{0} \geq$ $1-p$ and $1<\lambda_{0}<1-p$. Let the former hold. Then $s \leq \sigma_{1}<\sigma_{2}<\ldots<\sigma_{m}$ implies that

$$
\begin{aligned}
\sum_{i=1}^{m} q_{i}(1-p)^{s-\sigma_{i}}>\sum_{i=1}^{m} q_{i} \lambda_{0}^{s-\sigma_{i}} & =\left(\lambda_{0}^{s}-p\right)\left(\lambda_{0}-1\right)^{k} \\
& >\left[(1-p)^{s}-p\right](-p)^{k} \geq(1-p)(-p)^{k}
\end{aligned}
$$

which is a contradiction to our assumption. Hence the latter holds. Consequently,

$$
\begin{aligned}
\sum_{i=1}^{m} q_{i}(1-p)^{s-\sigma_{i}} \leq \sum_{i=1}^{m} q_{i} \lambda_{0}^{s-\sigma_{i}} & =\left(\lambda_{0}^{s}-p\right)\left(\lambda_{0}-1\right)^{k} \\
& <\left(\lambda_{0}^{s}-p\right)(-p)^{k} \\
& <\left[(1-p)^{s}-p\right](-p)^{k}
\end{aligned}
$$

gives a contradiction. By Proposition A, the proof is complete.

Theorem 3.5. $\left(E_{1}\right)$ admits a positive solution which tends to $+\infty$ as $n \rightarrow \infty$.

Proof. Setting

$$
G(\lambda)=\left(1-p \lambda^{-s}\right)(\lambda-1)^{k}-\sum_{i=1}^{m} q_{i} \lambda^{-\sigma_{i}}
$$

we may note that $G(1)<0$. Further,

$$
G(\lambda)=\lambda^{k}\left(1-p \lambda^{-s}\right)\left(1-\lambda^{-1}\right)^{k}-\sum_{i=1}^{m} q_{i} \lambda^{-\sigma_{i}}
$$

implies that $G(\lambda) \rightarrow \infty$ as $\lambda \rightarrow \infty$. Hence, there exists $\lambda_{0}>1$ such that $G\left(\lambda_{0}\right)=0$ and hence $\left(E_{1}\right)$ has a solution of the type $y(n)=\left\{\lambda_{0}^{n}\right\}$ which tends to $+\infty$ as $n \rightarrow \infty$. 
Remark 3.6. If we restrict our attention to $p \leq 0, s \leq \sigma_{1}<\sigma_{2}<\ldots<\sigma_{m}$ and $\sum_{i=1}^{m} q_{i} \geq 1-p$, then $\left(E_{1}\right)$ doesn't admit a positive solution of type $\left\{a^{n}\right\}, 0<a<1$.

Indeed, if $0<\lambda<1$, then (3.2) takes the form

$$
\begin{aligned}
G(\lambda) & =\lambda^{-s}\left[\left(\lambda^{s}-p\right)(\lambda-1)^{k}-\sum_{i=1}^{m} q_{i} \lambda^{s-\sigma_{i}}\right] \\
& <0, \text { if } k \text { is odd. }
\end{aligned}
$$

On the other hand, when $k$ is even

$$
G(\lambda)<\lambda^{-s}\left[(1-p)-\sum_{i=1}^{m} q_{i}\right] \leq 0
$$

where we have used the fact that $\lambda^{s}-p<1-p$ and $\left(\lambda^{s}-p\right)(\lambda-1)^{k}<1-p$. Hence $G(\lambda) \neq 0$, for $0<\lambda<1$. Thus our assertion follows.

Theorem 3.7. Let $p<-1$. Then $\left(E_{1}\right)$ admits an oscillatory solution in the following cases:

(i) $s$ and $\sigma_{i}, i=1,2, \ldots, m$ are odd integers when $k$ is odd

(ii) $s$ is odd and $\sigma_{i}, i=1,2, \ldots, m$ are even integers when $k$ is even.

Proof. Indeed, for Case(i)

$$
\begin{aligned}
G(-1) & =(-1)^{s}\left[(-1)^{s}-p\right](-1-1)^{k}-\sum_{i=1}^{m} q_{i}(-1)^{-\sigma_{i}} \\
& =-(-1-p)(-2)^{k}+\sum_{i=1}^{m} q_{i}=(p+1) 2^{k}+\sum_{i=1}^{m} q_{i}>0
\end{aligned}
$$

and from (3.3), it follows that $G(\lambda) \rightarrow-\infty$ as $\lambda \rightarrow-\infty$. Hence, there exists $\lambda_{0}<-1$ such that $G\left(\lambda_{0}\right)=0$ and $y(n)=\left\{\lambda_{0}^{n}\right\}$ is an oscillatory solution of $\left(E_{1}\right)$.

For Case(ii), it is easy to verify that $G(-1)<0$. From (3.3), it follows that $G(\lambda) \rightarrow$ $\infty$ as $\lambda \rightarrow-\infty$. Consequently, there exists $\lambda_{0}<-1$ such that $G\left(\lambda_{0}\right)=0$ and $y(n)=$ $\left\{\lambda_{0}^{n}\right\}$ is an oscillatory solution of $\left(E_{1}\right)$. This completes the proof.

Theorem 3.8. Let $p \geq 0$ and $k$ be even. If

$$
\sum_{i=1}^{m}\left(\sigma_{i}\right)^{-\sigma_{i}}\left(k+\sigma_{i}\right)^{k+\sigma_{i}} q_{i}<k^{k}
$$

then $\left(E_{1}\right)$ is oscillatory. 
Proof. Suppose on the contrary that $\lambda>0, \lambda \neq 1$ is a root of $\left(C_{1}\right)$. In what follows, $G(\lambda)$ has a positive root in $(0,1) \cup(1, \infty)$, so (3.2) can be written as

$$
G(\lambda)=(\lambda-1)^{k}\left[\left(1-p \lambda^{-s}\right)-\sum_{i=1}^{m} \frac{q_{i} \lambda^{-\sigma_{i}}}{(\lambda-1)^{k}}\right] .
$$

Setting $f(\lambda)=\frac{\lambda^{-\sigma_{i}}}{(\lambda-1)^{k}}$, we may note that $\min f(\lambda)$ attains at $\lambda=\frac{\sigma_{i}}{\left(k+\sigma_{i}\right)}$. Hence $\min f(\lambda)<f(\lambda)$ implies that

$$
\begin{aligned}
G(\lambda) & <(\lambda-1)^{k}\left[1-\sum_{i=1}^{m} q_{i} \min f(\lambda)\right] \\
& =(\lambda-1)^{k}\left[1-\sum_{i=1}^{m}\left(\sigma_{i}\right)^{-\sigma_{i}}\left(k+\sigma_{i}\right)^{k+\sigma_{i}} q_{i} k^{-k}\right] \\
& <0
\end{aligned}
$$

due to (3.4). Because $k$ is odd, $\left(C_{1}\right)$ has no positive roots in $(0,1) \cup(1, \infty)$. By Proposition $\mathrm{A},\left(E_{1}\right)$ is oscillatory.

Theorem 3.9. Let $p<0$ and $k$ be odd. If

$$
\sum_{i=1}^{m}\left(\sigma_{i}\right)^{-\sigma_{i}}\left(k+\sigma_{i}\right)^{k+\sigma_{i}} q_{i}<(1-p) k^{k},
$$

then $\left(E_{1}\right)$ is oscillatory.

Proof. Proceeding as in the proof of Theorem 3.8, we have a positive root in $(0,1) \cup$ $(1, \infty)$. Assume that $0<\lambda<1$. From (3.2), it follows that

$$
G(\lambda)=-(1-\lambda)^{k}\left[\left(1-p \lambda^{-s}\right)+\sum_{i=1}^{m} \frac{q_{i} \lambda^{-\sigma_{i}}}{(\lambda-1)^{k}}\right]
$$

Using the same type of reasoning as in Theorem 2.8, the above relation becomes

$$
\begin{aligned}
G(\lambda) & <-(1-\lambda)^{k}\left[1+\sum_{i=1}^{m}\left(\sigma_{i}\right)^{-\sigma_{i}}\left(k+\sigma_{i}\right)^{k+\sigma_{i}} q_{i} k^{-k}\right] \\
& <0 .
\end{aligned}
$$

Hence, $0<\lambda<1$ is not a root of $\left(C_{1}\right)$.

Next, we suppose that $\lambda>1$. Then $1-p \lambda^{-s}<1-p$ implies that $0<\lambda<1$. From (3.2), it follows that

$$
G(\lambda)<(\lambda-1)^{k}\left[(1-p)-\sum_{i=1}^{m} \frac{q_{i} \lambda^{-\sigma_{i}}}{(\lambda-1)^{k}}\right]
$$


and hence proceeding as in the proof of Theorem 3.8, we obtain

$$
\begin{aligned}
G(\lambda) & <(\lambda-1)^{k}\left[1-p-\sum_{i=1}^{m}\left(\sigma_{i}\right)^{-\sigma_{i}}\left(k+\sigma_{i}\right)^{k+\sigma_{i}} q_{i} k^{-k}\right] \\
& <0
\end{aligned}
$$

if and only if (3.5) holds. Hence by Proposition A, $\left(E_{1}\right)$ is oscillatory. This completes the proof.

\section{Application (Linearized Oscillation)}

We need the following lemma to prove our main result.

Lemma 4.1. Assume that (1.7) is oscillatory. Then there exists an $\epsilon_{0}>0$ such that for each $\epsilon \in\left[0, \epsilon_{0}\right]$, every solution of

$$
\triangle^{k}\left[u(n)-\left(p_{0}+2 \epsilon\right) u(n-s)\right]+\sum_{i=1}^{m}\left(q_{i}^{0}-\epsilon\right) u\left(n-\sigma_{i}\right)=0
$$

oscillates, if $k$ is odd or even and $\sigma_{i}>s, i=1,2, \ldots, m$.

Proof. Since (1.7) is oscillatory, then its characteristic equation

$$
F(\lambda)=\left(1-p_{0} \lambda^{-s}\right)(\lambda-1)^{k}+\sum_{i=1}^{m} q_{i} \lambda^{-\sigma_{i}}=0
$$

has no positive real roots.

Let $\lambda>0$, but $\neq 1$ be a root of $F(\lambda)$. Let $0<\epsilon^{*}<\frac{q_{i}^{0}}{3}$, for $i=1,2, \ldots, m$. Set

$$
G^{*}(\lambda)=2 \epsilon^{*} \lambda^{-s}(\lambda-1)^{k}+\epsilon^{*} \sum_{i=1}^{m} \lambda^{-\sigma_{i}}
$$

Thus

$$
F(\lambda)-G^{*}(\lambda)=\left[1-\left(p_{0}+2 \epsilon\right) \lambda^{-s}\right](\lambda-1)^{k}+\sum_{i=1}^{m}\left(q_{i}^{0}-\epsilon\right) \lambda^{-\sigma_{i}}
$$

and hence $F(\lambda)-G^{*}(\lambda) \rightarrow \infty$ as $\lambda \rightarrow \infty$. It is possible to find $\lambda_{0}>0$ such that $\lambda \geq \lambda_{0}$ implies that $F(\lambda)-G^{*}(\lambda)>\frac{m}{2}$, where $m=\inf _{\lambda>0} F(\lambda)>0$. Suppose that

$$
\epsilon_{0}=\min \left\{\epsilon^{*}, \frac{m \lambda_{0}^{s}}{2 m+4\left(\lambda_{0}-1\right)^{k}}, \frac{m}{2 \sum_{i=1}^{m} \lambda_{0}^{-\sigma_{i}}}\right\}
$$


Let $0<\epsilon \leq \epsilon_{0}$. The characteristic equation of (4.1) is given by

$$
G_{\epsilon}(\lambda)=\left[1-\left(p_{0}+2 \epsilon\right) \lambda^{-s}\right](\lambda-1)^{k}+\sum_{i=1}^{m}\left(q_{i}^{0}-\epsilon\right) \lambda^{-\sigma_{i}}=0 .
$$

In view of Proposition A and to complete the proof of the lemma, it is sufficient to show that (4.2) doesn't admit a positive root. Let $k$ be even. Then for $\lambda \geq \lambda_{0}$

$$
\begin{aligned}
G_{\epsilon}(\lambda) & =F(\lambda)-\epsilon\left[2 \lambda^{-s}(\lambda-1)^{k}+\sum_{i=1}^{m} \lambda^{-\sigma_{i}}\right] \\
& \geq F(\lambda)-\epsilon^{*}\left[2 \lambda^{-s}(\lambda-1)^{k}+\sum_{i=1}^{m} \lambda^{-\sigma_{i}}\right] \\
& \geq F(\lambda)-G^{*}(\lambda)>\frac{m}{2} .
\end{aligned}
$$

On the other hand, if $\lambda \in\left(0, \lambda_{0}\right)$, then $\sigma_{i}>s$ implies

$$
\begin{aligned}
G_{\epsilon}(\lambda) & \geq F(\lambda)-\epsilon\left[2 \lambda^{-s}(\lambda-1)^{k}+m \lambda^{-s}\right] \\
& \geq F(\lambda)-\epsilon\left[2 \lambda^{-s}\left(\lambda_{0}-1\right)^{k}+m \lambda_{0}^{-s}\right]>\frac{m}{2} .
\end{aligned}
$$

Hence, $0<\lambda_{0} \neq 1$ is not a root of (4.2).

Next, we assume that $k$ is odd. For $\lambda \geq \lambda_{0}>1$,

$$
\begin{aligned}
G_{\epsilon}(\lambda) & \geq F(\lambda)-\epsilon^{*}\left[2 \lambda^{-s}(\lambda-1)^{k}+\sum_{i=1}^{m} \lambda^{-\sigma_{i}}\right] \\
& \geq F(\lambda)-G^{*}(\lambda)>\frac{m}{2} .
\end{aligned}
$$

If $\lambda \in\left(0, \lambda_{0}\right)$ and $\lambda_{0}<1$, then

$$
\begin{aligned}
G_{\epsilon}(\lambda) & >F(\lambda)-\epsilon \sum_{i=1}^{m} \lambda^{-\sigma_{i}} \\
& =F(\lambda)-\epsilon_{0} \sum_{i=1}^{m} \lambda_{0}^{-\sigma_{i}}>m-\frac{m}{2}=\frac{m}{2} .
\end{aligned}
$$

Thus, $G_{\epsilon}(\lambda)>0$ for all $\lambda>0$. This completes the proof of the lemma.

Theorem 4.2. Suppose that (1.7) is oscillatory. Then every unbounded solution of (1.2) oscillates, if $k$ is odd or even and $\sigma_{i}>s$, for $i=1,2, \ldots, m$.

Proof. Let $y(n)$ be an unbounded nonoscillatory solution of (1.2) on $\left[n_{1}, \infty\right)$, for $n_{1} \geq$ $n_{0}+\rho$ such that $y(n)>0$, for $n \geq n_{1}$. The case $y(n)<0$, for $n \geq n_{1}$ may similarly be dealt with. For $n \geq n_{2}>n_{1}+\rho$, set

$$
z(n)=y(n)-p(n) g(y(n-s)) .
$$


Then we obtain

$$
\triangle^{k} z(n)=-\sum_{i=1}^{m} q_{i}(n) h_{i}\left(y\left(n-\sigma_{i}\right)\right)<0 .
$$

Therefore, $\triangle^{j} z(n)$ is monotonic on $\left[n_{3}, \infty\right)$, for $j=n-1, n-2, \ldots, 1,0$. If $\triangle^{k-1} z(n)>$ 0 , for $n \geq n_{3}$, then it follows from (4.3) that

$$
\begin{aligned}
\sum_{t=n_{3}}^{n-1} \sum_{i=1}^{m} q_{i}(t) h_{i}\left(y\left(t-\sigma_{i}\right)\right) & =-\triangle^{k-1} z(n)+\triangle^{k-1} z\left(n_{3}\right) \\
& <\triangle^{k-1} z\left(n_{3}\right) .
\end{aligned}
$$

Since $\triangle y(n)>0$, then the above inequality yields that

$$
\sum_{t=n_{3}}^{n-1} \sum_{i=1}^{m} h_{i}^{0} q_{i}(t)<\triangle^{k-1} z\left(n_{3}\right)
$$

due to (1.6). Hence

$$
\sum_{t=n_{3}}^{\infty} \sum_{i=1}^{m} h_{i}^{0} q_{i}(t)<\infty
$$

a contradiction to the fact that

$$
\sum_{t=n_{3}}^{\infty} \sum_{i=1}^{m} h_{i}^{0} q_{i}(t)=\infty
$$

due to (1.4), because $q_{i}^{0}>0$. Ultimately, $\triangle^{k-1} z(n)<0$, for $n \geq n_{3}$. Thus $\triangle^{j} z(n)<0$, for $j=0,1,2, \ldots,(k-2), k \geq 2$. If $k=1$, then $\triangle z(n)<0$, for $n \geq n_{4}>n_{3}$ and proceeding as above we may show that $z(n)<0$, for $n \geq n_{4}$. Let $\lim _{n \rightarrow \infty} z(n)=\mu$, $\mu \in(-\infty, 0)$. Then summing (4.3) for $k=1$, we arrive at a contradiction. Thus $\lim _{n \rightarrow \infty} z(n)=-\infty$, for $k \geq 1$. For $n \geq n_{4}$, Eq.(1.2) may be written as

$$
\Delta^{k}(y(n)-P(n) y(n-s))+\sum_{i=1}^{\infty} Q_{i}(n) y\left(n-\sigma_{i}\right)=0
$$

that is,

$$
\Delta^{k} Z(n)+\sum_{i=1}^{\infty} Q_{i}(n) y\left(n-\sigma_{i}\right)=0
$$

where $Z(n)=y(n)-P(n) y(n-s), P(n)=p(n) \frac{g(y(n-s))}{y(n-s)}$ and $Q_{i}(n)=q_{i}(n) \frac{h_{i}\left(y\left(n-\sigma_{i}\right)\right)}{y\left(n-\sigma_{i}\right)}$. Using (1.3) and (1.5), we may note that $\limsup _{n \rightarrow \infty} P(n) \leq$ 
$\limsup _{n \rightarrow \infty} p(n) \cdot \lim _{n \rightarrow \infty} \frac{g(y(n-s))}{y(n-s)}=p_{0}$ and $\limsup _{n \rightarrow \infty} P(n) \geq \limsup _{n \rightarrow \infty} p(n)=p_{0}$. Hence $\limsup P(n)=p_{0}$. Summing (4.4) $k$-times from $n_{4}$ to $n-1\left(n>n_{4}+1\right)$, we obtain $n \rightarrow \infty$

$$
Z(n)+\frac{1}{(k-1) !} \sum_{j=n_{4}}^{n-1}(n-j-1)^{(k-1)}\left[\sum_{i=1}^{\infty} Q_{i}(j) y\left(j-\sigma_{i}\right)\right]<0
$$

where $\beta^{(\nu)}=0$ if $\nu>\beta$, and $=1$ if $\nu=0$; otherwise (see, e.g., [5]),

$$
\beta^{(\nu)}=\beta(\beta-1) \ldots(\beta-\nu+1) .
$$

From the last inequality, it follows that

$$
\begin{aligned}
& y(n) \\
& >\frac{1}{P(n+s)}\left[y(n+s)+\frac{1}{(k-1) !} \sum_{j=n_{4}}^{n+s-1}(n+s-j-1)^{(k-1)} \sum_{i=1}^{\infty} Q_{i}(j) y\left(j-\sigma_{i}\right)\right] .
\end{aligned}
$$

From Lemma 4.1, it follows that there exists an $\epsilon_{0}>0$ such that for each $\epsilon$ with $0 \leq$ $\epsilon \leq \epsilon_{0}$, every solution of (4.1) oscillates if $k$ is odd or even and $\sigma_{i}>s, i=1,2, \ldots, m$. In what follows, we show that 4.1 admits a positive solution leading to a contradiction which completes the proof of the theorem. Let $N_{1}, N_{2}>0$ be such that $P(n)<p_{0}+\epsilon$, for $n \geq N_{1}$ and $Q_{i}(n)>\left(q_{i}^{0}-\epsilon\right)$, for $n \geq N_{2}$. Let $N_{0}=\max \left\{n_{4}, N_{1}, N_{2}\right\}$. Choose $1<\beta<1+\frac{\epsilon}{p_{0}+\epsilon}$. Thus for $n \geq N_{0}, P(n)<\left(p_{0}+2 \epsilon\right) / \beta$ and hence (4.5) yields

$$
\begin{aligned}
& y(n) \\
& >\frac{\beta}{p_{0}+2 \epsilon}\left[y(n+s)+\frac{1}{(k-1) !} \sum_{j=N_{0}}^{n+s-1}(n+s-j-1)^{(k-1)} \sum_{i=1}^{\infty} Q_{i}(j) y\left(j-\sigma_{i}\right)\right] .
\end{aligned}
$$

Let $X=\ell_{N_{0}}^{\infty}$ be the Banach space of all real valued functions defined on $\left[N_{0}-\sigma_{m}-s, \mathbb{R}\right)$ with the usual sup norm. Let us define

$$
B=\left\{x \in X: 0<x(n) \leq 1, n \geq N_{0}-\sigma_{m}-s\right\}
$$

Clearly, $B$ is a closed, bounded and convex subset of $X$. For $x \in B$, we define $T: B \rightarrow$ 
$X$ as follows:

$$
(T x)(n)=\left\{\begin{array}{l}
\frac{(T x)\left(N_{0}\right), \quad N_{0}-\sigma_{m}-s \leq n \leq N_{0}}{y(n)}\left[y(n+s) x(n+s)+\frac{1}{(k-1) !} \sum_{j=N_{0}}^{n+s-1}(n+s-j-1)^{(k-1)}\right. \\
\left.\quad \times \sum_{i=1}^{m}\left(q_{i}^{0}-\epsilon\right) x\left(j-\sigma_{i}\right) y\left(j-\sigma_{i}\right)\right], \quad n \geq N_{0} .
\end{array}\right.
$$

Thus for $n \geq N_{0}-\sigma_{m}-s, T x(n)>0$ and $(T x)(n)$

$$
\begin{aligned}
& \leq \frac{\left(p_{0}+2 \epsilon\right)^{-1}}{y(n)}\left[y(n+s)+\frac{1}{(k-1) !} \sum_{j=N_{0}}^{n+s-1}(n+s-j-1)^{(k-1)} \sum_{i=1}^{m}\left(q_{i}^{0}-\epsilon\right) y\left(j-\sigma_{i}\right)\right] \\
& <\frac{1}{\beta}<1 .
\end{aligned}
$$

Thus $T: B \rightarrow B$. Further, for $x_{1}, x_{2} \in B$,

$$
\left|T x_{1}(n)-T x_{2}(n)\right|=\left\{\begin{array}{l}
\left|\left(T x_{1}\right)\left(N_{0}\right)-\left(T x_{2}\right)\left(N_{0}\right)\right|, \quad N_{0}-\sigma_{m}-s \leq n \leq N_{0} \\
\frac{\left(p_{0}+2 \epsilon\right)^{-1}}{y(n)} \mid y(n+s)\left\{x_{1}(n+s)-x_{2}(n+s)\right\}+ \\
\frac{1}{(k-1) !} \sum_{j=N_{0}}^{n+s-1}(n+s-j-1)^{(k-1)} \times \\
\sum_{i=1}^{m}\left(q_{i}^{0}-\epsilon\right) y\left(j-\sigma_{i}\right)\left\{x_{1}\left(j-\sigma_{i}\right)-x_{2}\left(j-\sigma_{i}\right)\right\} \mid, \quad n \geq N_{0} .
\end{array}\right.
$$

Thus, for $n \geq N_{0}-\sigma_{m}-s$

$$
\begin{gathered}
\left|T x_{1}(n)-T x_{2}(n)\right| \leq \frac{\left\|x_{1}-x_{2}\right\|}{\left(p_{0}+2 \epsilon\right) y(n)}\left[y(n+s)+\frac{1}{(k-1) !} \sum_{j=N_{0}}^{n+s-1}(n+s-j-1)^{(k-1)} \times\right. \\
\left.\sum_{i=1}^{m}\left(q_{i}^{0}-\epsilon\right) y\left(j-\sigma_{i}\right)\right]<\frac{1}{\beta}\left\|x_{1}-x_{2}\right\| .
\end{gathered}
$$

Hence

$$
\left\|T x_{1}(n)-T x_{2}(n)\right\| \leq \frac{1}{\beta}\left\|x_{1}-x_{2}\right\|
$$


implies that $T$ is a contraction mapping. Therefore, $T$ has a unique fixed point in $B$, that is, there exists $x_{0} \in B$ such that $T x_{0}(n)=x_{0}(n)$, that is,

$$
x_{0}(n)=\left\{\begin{array}{l}
x_{0}\left(N_{0}\right), \quad N_{0}-\sigma_{m}-s \leq n \leq N_{0} \\
\frac{\left(p_{0}+2 \epsilon\right)^{-1}}{y(n)}\left[y(n+s) x_{0}(n+s)+\frac{1}{(k-1) !} \sum_{j=N_{0}}^{n+s-1}(n+s-j-1)^{(k-1)}\right. \\
\left.\times \sum_{i=1}^{m}\left(q_{i}^{0}-\epsilon\right) x_{0}\left(j-\sigma_{i}\right) y\left(j-\sigma_{i}\right)\right], \quad n \geq N_{0} .
\end{array}\right.
$$

Set $w(n)=y(n) x_{0}(n)$, for $n \geq N_{0}-\sigma_{m}-s$. We note that $w(n)>0$, where

$$
\begin{aligned}
& w(n) \\
& =\left(p_{0}+2 \epsilon\right)^{-1}\left[w(n+s)+\frac{1}{(k-1) !} \sum_{j=N_{0}}^{n+s-1}(n+s-j-1)^{(k-1)} \sum_{i=1}^{m}\left(q_{i}^{0}-\epsilon\right) w\left(j-\sigma_{i}\right)\right],
\end{aligned}
$$

for $n \geq N_{0}$. Thus

$$
\begin{aligned}
w(n-s) & =\left(p_{0}+2 \epsilon\right)^{-1} \times \\
& {\left[w(n)+\frac{1}{(k-1) !} \sum_{j=N_{0}}^{n-1}(n-j-1)^{(k-1)} \sum_{i=1}^{m}\left(q_{i}^{0}-\epsilon\right) w\left(j-\sigma_{i}\right)\right] }
\end{aligned}
$$

and hence

$$
\triangle^{k}\left[w(n)-\left(p_{0}+2 \epsilon\right) w(n-s)\right]+\sum_{i=1}^{m}\left(q_{i}^{0}-\epsilon\right) w\left(j-\sigma_{i}\right)=0 .
$$

Therefore, $w(n)$ is a positive solution of (4.1), a contradiction. This completes the proof.

\section{Example and Discussion}

Example 5.1. Consider

$$
\begin{aligned}
\triangle^{2}\left[y(n)-\left(1+\frac{1}{n}\right) g(y(n-1))\right] & +\left(1-\frac{1}{n}\right) h_{1}(y(n-2))+ \\
& +\left(2-\frac{1}{n}\right) h_{2}(y(n-3))=0, n \geq 4,
\end{aligned}
$$


where $k=2, g(u)=h_{1}(u)=h_{2}(u)=u\left(1+e^{-|u|}\right)$, for $u \in \mathbb{R}, s=1, \sigma_{1}=1, \sigma_{2}=3$. Clearly, $g, h_{1}$ and $h_{2}$ satisfy the conditions (1.5) and (1.6). Further, $\lim _{n \rightarrow \infty} p(n)=1$, $\lim _{n \rightarrow \infty} q_{1}(n)=q_{1}^{0}=1$ and $\lim _{n \rightarrow \infty} q_{2}(n)=q_{2}^{0}=2$ imply that the limiting equation (1.7) takes the form

$$
\triangle^{2}[x(n)-x(n-1)]+x(n-2)+2 x(n-3)=0
$$

and the characteristic equation associated with it is given by

$$
F(\lambda) \equiv\left(1-\lambda^{-1}\right)(\lambda-1)^{2}+\lambda^{-2}+2 \lambda^{-1}=0 .
$$

Since $q_{1}^{0}+q_{2}^{0}>1$, then (5.3) has no positive roots by Theorem 2.3. Hence (5.2) is oscillatory. Consequently, every solution of (5.1) oscillates by Theorem 4.2.

Explicitly, we connect $(2 m+3)$ parameters in our discussion except Theorems 2.7, 3.7 and 3.8, where the delay terms are free from any restrictions. It is left to the interested readers to formulate more general results in other ranges of $p$ for any $k$.

\section{References}

[1] B. Baculikova, J. Dzurina; Oscillation theorems for higher order neutral differential equations, Appl. Math. Compu., 219(2012), pp. 3769-3778.

[2] B. Baculikova, J. R. Graef, J. Dzurina; On the oscillation higher order delay differential equations, Nonlinear Oscillations, 15(2012), pp. 13-24.

[3] S. S. Cheng, Y. Z. Lin; Complete characterization of an oscillatory neutral difference equation, J. Math. Anal. Appl., 221(1998), pp. 73-91.

[4] I. Gyori, G. Ladas; Oscillation Theory for Delay Differential Equations with Applications, Clarendon Press, Oxford,1991.

[5] W. G. Kelley, A. C. Peterson; Difference Equations: An Introduction with Applications, Acadenic Press, New York 1991.

[6] K. Kowalski, W. H. Steeb; Nonlinear Dynamical Systems and Carleman Linearization, World Scientific, Singapore, 1991.

[7] G. Ladas, C. Qian; Linearized oscillation for odd order neutral delay differential equations, J. Diff. Equ., 88(1990), pp. 238-247.

[8] G. Ladas, C. Qian; Linearized oscillation for even order neutral differential equations, J. Math. Anal. Appl., 159(1991), pp. 237-250. 
[9] Y. Z. Lin, S. S. Cheng; Complete characterizations of a class of oscillatory difference equations, J. Diff. Equ. Appl., 2(1996), pp. 301-313.

[10] N. Parhi; Linearized oscillation for higher order neutral differential equations, Proc. Indian Acad. Sci., 106(1996), pp.169-176.

[11] N. Parhi, A. K. Tripathy; Oscillation of a class of nonlinear neutral difference equations of higher order, J. Math. Anal. Appl, 284(2003), pp. 756-774. 